\title{
Design of Behavior Change Environment with Interactive Signage Having Active Talk Function
}

\author{
Zhihua Zhang $^{1}$, Yutaka Arakawa ${ }^{1,2}$, Harri Oinas-kukkonen ${ }^{3}$ \\ ${ }^{1}$ Nara Institute of Science and Technology, Ikoma, Nara 630-0192, JAPAN \\ E-mail: zhang.zhihua.yn2@is.naist.jp, ara@is.naist.jp, harri.oinas-kukkonen@oulu.fi \\ 2 JST PRESTO \\ ${ }^{3}$ University of Oulu, Finland
}

\begin{abstract}
In this paper, we construct an interactive signage system, which is able to actively talk to users who are passing the signage system and at the same time tries to induce behavioral changes through visual and auditory stimulation. On top of our previous feasibility study, we re-design our proposed system based on the persuasive system design (PSD) model and the behavior change support system (BCSS) theory. The new design consists of 4 parts: recognizing (identifying and classifying users), executing (sending triggers and inducing behavior change), reviewing (recording users' reaction), and feedback (keeping or improving users' motivation). To track users' status and performance, we use the smartphone and the smartwatch to collect users' bio-data (e.g. heart rate, number of steps) and users' location. To send user triggers continuously without being interrupted by notifications from other applications, we set the interactive signage in the daily action line of users and present information to users when they pass the signage. In order to make the system more persuasive, we pick up eight features from the 28-feature list of PSD and apply them to our design.
\end{abstract}

Index Terms-behavior change, interactive signage, beacon, environment side, behavior change support system, persuasive system design

\section{INTRODUCTION}

With the progress of information and communication technology in recent years, various Internet of Things (IoT) devices are spreading in our daily lives. At the same time, due to the sophistication of Artificial Intelligence (AI) technology, we can recognize people's behavior with better accuracy and make the human-computer dialogue more natural. By using these powerful IoT and AI technologies, many research and developments related to behavior change have been conducted. Behavior change is also often called persuasion, meaning "reinforcing, forming or altering a users attitude or behavior or an act of complying without coercion or deception" [1]. In the medical field, a classic way for inducing a behavior change is a consultation to patients that tries to show the importance for changing and future risk of not changing, then ask them to record their personal data like weight and nutrition they ingested every day.

Since people are easy to forget recording and are unwilling to change their daily life, this method faces the challenge of discontinuity. To cope with this problem, a lot of research and development to promote behavior change by using information technology have shown up. For instance, Sankaran et al. have proposed a telerehabilitation application which induces Coronary Artery Disease (CAD) patients to comply with the treatment plan and prevent disease recurrence [2]. In the management of smoking cessation, hazardous drinking, obesity, diabetes, asthma, tinnitus, stress, depression, and insomnia, positive results have also been obtained [3]. Therefore, the information-technology-based method enabling behavior change is also considered as Digital Medicine and have been extensively studied in Europe and in the U.S. [4].

Not only in the medical field but also many examples exist in other areas. For example, Pokémon Go is an argument reality game and it has been shown that Pokémon Go can serve in increasing players' physical activity [5]. Consolvo et al. developed an application, where a user's physical activity leads to a more beautiful and growing virtual garden [6]. Some researchers have used additional sensors and equipment for promoting behavior change such as nurturing a healthy lifestyle like, exercising more or drinking more water. For example, Fortmann et al. developed a bracelet with embedded LEDs to improve users' drinking behavior [7].

Most of these developments are designed to help people with improving their lifestyle or maintaining their improvement. From the viewpoint of cost and information reachability, the information system of the many studies were developed as mobile applications. Nowadays, people practically always carry their own smartphones, that makes it possible for researchers and developers to collect personal data and sense the environmental context through the mobile application. However, this approach has a problem with sending triggers. The common way for sending triggers to users is a push notification. Namely, users nowadays receive a huge number of notifications from various apps that it all too often interrupts the users and it may diminish noticing triggers from the behavior change apps. Also, when users end-up turning their phones into a silent mode in which they do not receive push messages, many of the message may remain unnoticed. Moreover, the mobile-application-based methods often used in current systems like reminder, social competition, etc., may have to overcome the weak points which affect the quality of behavior change apps. Even if an app may induce behavior change successfully, it is hard to maintain the effect due to the limitations of the methods based on mobile apps. Therefore, alternative approaches are needed for improving the efficiency of digital interventions and both inducing behavior change and 
maintaining the result.

To resolve these problems of mobile application side, we propose here a design of a behavior change support system [1] environment. This technological environment means an enclosed place where people spend a long time (e.g. a company, a university or an elder care center) equipped with smart devices for inducing and/or maintaining behavior change. In our design, both the user side and environment side are included for analyzing a user's status and presenting userrelated information to the user. In the user side, we use the smartwatch and smartphone to collect user's personal data (e.g. heart rate, number of steps). In the environment side, we use the interactive signage with an active talk function and deploy the signage in the daily action line of users. By using the active talk function, we can send visual and auditory stimulation to users. By setting the device in the daily action line, it can recognize the daily patterns of users and continuously send triggers to users. We also consider this environment as a small society. Therefore, the target of this environment is not only a single individual but also a group and we apply social support to this design with careful consideration of users' privacy and social relationship.

Based on the BCSS theory [1], there are four parts in our design: recognizing, executing, reviewing and providing feedback:

- The Recognizing part is for understanding users' needs, goals, past performance and current progress. According to the theory of goal setting, people with high selfefficacy set higher goals and have higher performance than people with low self-efficacy [8]. It is necessary to change the content of triggers based on the user's type. Therefore, in the recognizing part, we estimate the type of user based on user's performance and progress.

- The Executing part is the part where we send triggers to the user and try to induce a behavior change after we divide users into groups. The triggers contain userrelated information (e.g. name, weight) and social-related information (e.g. ranking of performance and contribution of other members).

- The Reviewing part is for checking users' progress.

- The Feedback part is for encouraging users and improving the motivation based on the result of the reviewing part.

By applying the design of behavior change environment, as long as people are staying in this environment, our system is expected to be able to send stimulation to users and promote behavior change continuously, so that we can improve the efficiency of digital intervention and the quality of behavior change.

The following sections describe the related work in Section 2 , the proposed behavior change environment design in Section 3 , and summary in Section 4.

\section{RElate WORK OF BCSS}

Behavior Change Support System (BCSS) Theory [1] is an extended version of Persuasive Systems Design (PSD) [9]. A behavior change support system is defined as "a sociotechnical information system with psychological and behavioral outcomes designed to form, alter or reinforce attitudes, behaviors or an act of complying without using coercion or deception" [1]. BCSS theory emphasizes the practical behavior change outcome and lists a set of software features for the BCSS. In our research, the BCSS theory is applied as the basic theory and guideline of designing our behavior change environment.

\section{A. Examples of BCSS}

With the progress of IoT and AI technology in recent years, the number of research and developments of inducing behavior change by using information technology have been increasing. These developments are mainly designed to help people with improving their life patterns or maintaining the result of improvement. And the approaches used for inducing behavior change are quite versatile.

Gamification has become popular recently since it can affect players' attitudes and behaviors through playfulness. Gamification may contain many attractive elements and it may succeed to keep players' motivation in using the application. In order to increase the activity of users, for instance, Consolvo et al. proposed an application embedding gamification in which the users' physical activity leads to a more beautiful and growing virtual garden [6]. Another famous BCSS example using gamification is Pokémon Go. It is an augmented reality exergame and has been shown to be able to serve as a BCSS [5]. The game can influence behavior on all three levels as it can form, alter, reinforce behaviors [1] and successfully add activity in players' life, enhance routines, exploration, increase physical activity, strengthen social bonds, lower social barriers, increase emotional expression and self-treatment [5].

Social support is another popular strategy used in current BCSSs. It isn't a single feature but including learning, comparison, competition, normative influence, facilitation, cooperation, and recognition [9]. Under the influence of social relationship and peer pressure, even people with low selfefficacy and low motivation may also try to achieve the goal set by the system. Some examples of BCSSs using social support include Jaques et al. who developed a web-based pairing application in which the two paired participants need to help each other with achieving their goals like drinking more water [10]. Luhanga et al. conducted a study in which they sought to promote behavior change in losing weight and balanced diet by using social competition among group members [11].

Also, devices given to users have been studied in the environment side. For example, Fortmann et al. developed a bracelet with embedded two kinds of LEDs to show the number of drinks during the day and the passed time since last time the user drank water with color-coded from green to red [7].

\section{B. Strategies Used in Current Systems and Their Weaknesses}

According to Matthews et al.'s research, self-monitoring, social comparison, reminder, competition, suggestion were the most used features in the system for promoting physical 
activity with mobile applications [12]. However, some of these strategies have weak points which may cause problems with continuity and efficiency.

Reminders are quite useful for helping users to remember tasks they need to do based on the setting. However, in Karppinen et al. study [13], it was also reported that reminders may make users depend on the reminder and harm users' own memory of the tasks. Participants in Stawarz et al.'s experiment who used reminders reported the lowest automaticity scores and tended to forget more often [14]. In our view, the main reason for the different performance of reminders in these studies was frequency. In Karppinen et al.'s research, a reminder message was sent to users if they didn't complete the weekly task and second reminder message if the situation didn't change. After that, no more reminder messages were sent. In Stawarz et al.'s experiment, participants received reminders during the whole period asking users to input the details of their meals. Based on these studies, reminders will still be a part of our design but with careful consideration of frequency.

In social support, competition is a widely used feature since it can stimulate users' motivation. However, it may also harm users' motivation if the system doesn't cover privacy and social relationship. Luhanga et al.'s system shared all the information including the failed tasks that users did which might affect the privacy and social relationship and increase the unwillingness of using the system [11]. Therefore, the privacy problem should be carefully considered when we apply social support to our design.

The self-monitoring is in many settings the most applied feature in promoting behavior change with mobile phones [12]. However, the limitation of self-monitoring is that the method for a great extent relies on the user. If users do not input or forget to input the data, then there will not be data to process. Current systems often combine the self-monitoring with the reminder as Stawarz et al. did [14]. This will cause a vicious circle that users would hardly form a daily habit of monitoring and logging themselves, which is against the purpose of behavior change. Therefore, self-monitoring should be more active in presenting the situation of the user instead of just waiting for the user to check and input data.

A fundamental problem with the current systems is that they are usually developed as mobile applications because users always carry their own smartphones, which makes it possible for researchers and developers to collect users' personal data and sensing the environmental context around users. By using smartphone applications, the cost can be reduced since there is no additional cost for additional devices. However, this approach has a problem with sending triggers. The common way in current systems for presenting information to users is using push notifications. But users nowadays receive so many notifications from various apps which can interrupt the messages from the behavior change apps. Also, if users change their phones to silent mode, it will be more difficult for them to notice the messages.

Therefore, alternative approaches are needed for improving

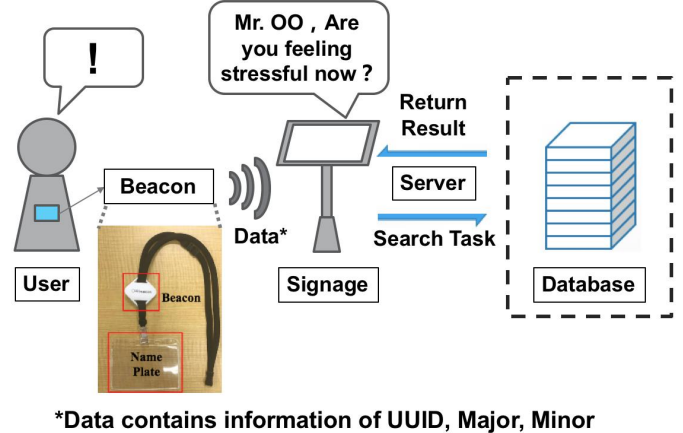

Fig. 1. System Configuration of Interactive Signage System

the efficiency of digital intervention and the quality of behavior change including inducing behavior change and maintaining the result.

\section{Interactive Signage with The Active Talk Function}

To induce a behavior change, information must be presented and the persuadee must pay attention to the arguments presented and comprehend it [9]. As we mentioned in Section 2.2 , using push notifications has the problem with sending triggers to the user. To resolve the problem, we developed an active talk function and installed it into an interactive signage. The system configuration of the interactive signage system is shown in Fig. 1. The interactive signage is a new type of digital signage equipped with a touch panel, sensors, and camera. It is becoming common in many public places like airport, company, and university. In this research, we used the iPad as an interactive signage since it allows users to control and respond to the contents through the touch panel. Also, it can visualize information and send the voice message. As a method to get users' attention, we developed a function called active talk function. In this method, when users pass around the interactive signage the system will judge whether to present the information or not. When the system decides to present the information, a voice and text message containing user-related information will be sent from the signage to stimulate the user's visual and audio sense. For identifying the user, we used BLE (Bluetooth Low Energy) beacon shown in Fig.1. It is tiny (coin size), light (8 grams), and easy to attach to a nameplate that the user always brings. By reading unique UUID (Universally Unique Identifier), major, minor of each BLE beacon, the system can identify the approaching user easier and faster compared with a camera-based user identification.

We did a 3-week survey experiment in our laboratory to investigate the answer of three questions: whether the interactive signage is able to attract the user's attention and curiosity or not; whether the interactive signage is able to collect data from users; whether the interactive signage is able to promote a behavior change. The interactive signage was deployed in 4 locations (Fig.2) which are in the daily action line of members. 15 participants joined this experiment and they were asked to carry the BLE beacon with their nameplate 


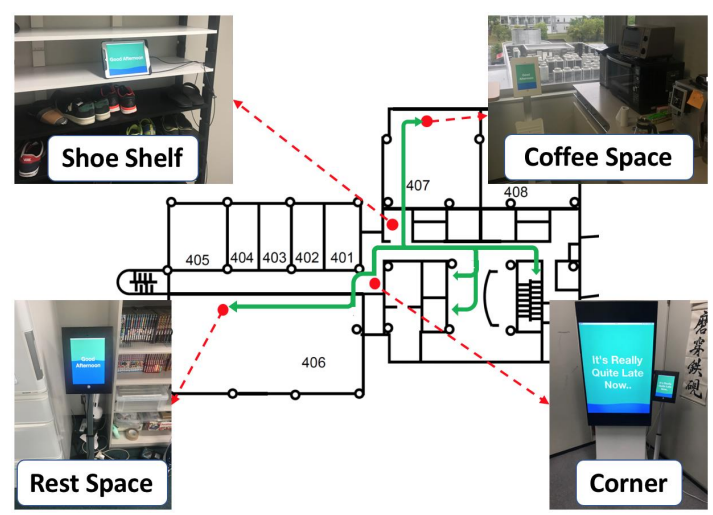

Fig. 2. Location of Interactive Signage

while staying in the laboratory during the experiment period. For this experiment, we prepared four kinds of scenarios for checking the status of participants and equipment. When the signage detected a passing participant by scanning the signal of the beacon, the system accessed a cloud server and searched for available tasks for the user. After finding an available task, the system presented the content of the task by a voice and text message. Users were able to respond to the task by pushing a button on the screen or inputting text.

As a result, even the lowest response rate of the user was nearly $70 \%$ [15]. This indicates that people respond to the inquiry from the signage with high probability and the interactive signage is able to collect information from the user. Also, according to the records of users' response, low labor and time cost behavior like checking the water level of a hot pot and the location of other members were induced successfully. However, even if we were able to attract users' attention, it doesn't actually achieved our goals. In fact, a more carefully designed system is needed for promoting more complex behavior to improve users' health condition and the environment. In our experimental study with the previous system [15], some of the users perceived the system to be troublesome and they did not want to check the statements of other users or the equipment like "How many people are there in lab now?" and "How much water left in the coffee maker?". In order to improve their motivation, it is important to provide feedback more suitable for them. We believe that especially feedback that lets people know how their behaviors would be affected, such as "how much energy has been saved thanks to one turning off the light", will improve users motivation and drive them to complete the tasks even when they require time and labor.

\section{Behavior Change Environment Design}

To design an environment for a behavior change support system, we first need to identify key behavioral patterns that foster behavioral change and maintenance and determine when and how the system should intervene. The behavior change environment here means an enclosed place where people spend a long time (e.g. a company, a university or an elder care

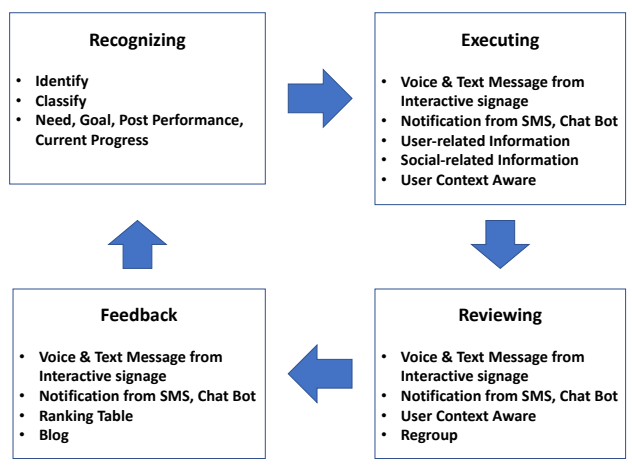

Fig. 3. Form of Proposed Behavior Change Environment

center) equipped with smart devices for inducing the change. In Alahäivälä et al.'s research, it was reported that most of the research on health behavior change was focusing on the reinforcing outcome and much less focused on forming or altering a behavior [16]. Due to individual differences, especially the differences of personal experiences, it is reasonable that altering or forming a behavior is much harder than reinforcing an existing one. However, in our research, altering or forming a behavior like promoting people to quit smoking or starting muscle training are also goals we want to achieve. Our design is focusing on the common issues that users meet with during in their daily lives in this kind of environment and trying to deal with addressing attitude, behavior change and an act of complying [1]. The common issues include improving health condition (e.g. diet, sleep, exercise, stress, drink water, etc.) and the environment (e.g. save energy, keep the environment clean, etc.). We believe that by using this system, many kinds of behaviors in daily life, such as replacing the water in the hot pot for other people and doing a health checkup while waiting for the coffee, among many others, can be induced.

\section{A. Form of Behavior Change Environment}

To achieve the goal of forming, altering or reinforcing users' attitude and behavior, we designed a behavior change environment consisting of 4 stages: recognizing, executing, reviewing, feedback, as shown in Fig.3.

The recognizing stage is mainly for identifying and classifying the user. The target user is identified by the signal of the carried BLE beacon and the application in their smartphones. In order to provide appropriate information to the user, we collect users' needs, goals, past performance, and current progress in this stage through the inquiry from the interactive signage. After collecting these data, the system classifies users and divides them into different groups.

The executing stage is the place where we try to induce a behavior change. In this stage, the interactive signage sends a voice and text message containing user-related information and the social-related information to the target user. If the user doesn't notice the message, another notification will be sent by the chatbot to the user. The user-related information means the content containing the user's personal data like name, gender, 
TABLE I

The Difference Between Previous System And New Design

\begin{tabular}{|c|c|c|c|}
\hline Phase & Previous System & Discussion & New Design \\
\hline Recognizing & - Use beacon & $\begin{array}{l}\text { - High self-efficacy people set higher } \\
\text { goals and have higher performance than } \\
\text { low self-efficacy people [8]. }\end{array}$ & $\begin{array}{l}\text { - Use beacon } \\
\text { - Classify user }\end{array}$ \\
\hline Executing & $\begin{array}{l}\text { - Send voice and text message } \\
\text { - Collect user's location } \\
\text { - User-related information }\end{array}$ & $\begin{array}{l}\text { - More data beside users' location is } \\
\text { needed for adjusting the timing and } \\
\text { frequency. } \\
\text { - For promoting a complex behavior } \\
\text { change, triggers need to be more attrac- } \\
\text { tive and impactive. }\end{array}$ & $\begin{array}{l}\text { - Send voice and text message } \\
\text { - Collect location and bio-data } \\
\text { - User-related information } \\
\text { - Use social-related information }\end{array}$ \\
\hline Reviewing & - Record the response to inquiry & $\begin{array}{l}\text { - In order to ensure the effectiveness of } \\
\text { the triggers, we need to track users' sta- } \\
\text { tus. For example, whether they finished } \\
\text { the tasks or not. } \\
\text { - After getting the triggers, users' attitude } \\
\text { or motivation might change. Therefore, } \\
\text { regrouping the users is needed. }\end{array}$ & $\begin{array}{l}\text { - Record the response to inquiry } \\
\text { - Track users' status (bio-data) } \\
\text { - Regroup users }\end{array}$ \\
\hline Feedback & - Send text message & $\begin{array}{l}\text { - According to the questionnaire after the } \\
\text { survey experiment, participants wish to } \\
\text { hear the voice message when they see } \\
\text { the text message. } \\
\text { - Feedback should be various based on } \\
\text { the type of content presented to the } \\
\text { user so that they can know how their } \\
\text { decisions affect the result. }\end{array}$ & $\begin{array}{l}\text { - Send voice and text message } \\
\text { - Show graph/chart } \\
\text { - Show ranking table } \\
\text { - Write blog/report }\end{array}$ \\
\hline
\end{tabular}

age, and weight. The social-related information means the content containing other user's information like their needs, goals, and progress. The content presented to the user is adjusted depending on the group of the user. For example, for the people who have high efficacy, the content focuses more on keeping the motivation of the user. For the low-efficacy people, the content will focus more on improving the motivation. To adjust the timing of presenting the information, we collect the user's bio-data (e.g. heart rate, number of steps) and location information.

In the reviewing stage, we record the user's reaction toward the information from the system. For the behavior related to movement and location, we estimate the user's reaction through the users' data. For the other behavioral patterns, we confirm the result through the response to the inquiry and notifications of the user. Based on the result, users will be regrouped again.

The feedback stage is for keeping and improving users' motivation. If the user responded to the message from the signage, the signage will send a voice or text message as a feedback. Also, the ranking table and a chart of the progress of the whole group is a part of the feedback. A blog (or report) about the progress of an individual or a group will be sent to the social media periodically. The content of feedback will also depend on the group of the user.

Table I shows the difference between the previouslydeveloped interactive signage and a newly-designed signage.

\section{B. Strategy and Theory Used in The Design}

Table II lists the features we apply to our design among 28 features listed in the BCSS theory [1], [9].

Reduction means to reduce a complex behavior into simple tasks which helps users perform the target behavior and it may increase the benefit/cost ratio of a behavior [1], [9]. In our design, all the behavior patterns are broken into smaller tasks and guide users to achieve the small tasks step by step. Another effort for the reduction in this design is that we let users to respond to the inquiry by pushing a single button and only a few cases need users to input a text.

Self-monitoring is widely used in the current systems because it can show the progress of users and the distance to their goals. In this design, we provide a way for the users to check their progress by themselves. Meanwhile, we also present their progress actively by the chatbot and signage so the low-motivation people can also be aware of their situation.

In the previous system, we used text praise as a feedback. But the participants reported that they want to hear the sound. Therefore, in this design, we apply both voice and text praise to our feedback part. Based on the result of reviewing, the tone and the content of the voice message will change.

The excessive use of reminders can harm users' memory to the tasks. Therefore, in this design, the reminders will be kept at a low level. Suggestion to some extent have a similar role as reminders but it persuades people in softer ways. However, it is still unclear that how would the suggestion affect the user's cognition. Investigating the effect of suggestion will be a part of this research. 
TABLE II

THE STRATEGIES APPLIED TO THE DESIGN

\begin{tabular}{l|l|l}
\hline Primary Task & Dialogue Support & Social Support \\
\hline \hline - Reduction & - Praise & - Comparison \\
- Self-monitoring & - Suggestion & - Facilitation \\
& - Reminder & - Competition \\
\hline
\end{tabular}

System users will have a greater motivation to perform the target behavior if they can compare their performance with the performance of other users, discern via the system that others are performing the behavior along with them or able to compete with other users [1], [9]. We consider the environment as a small society and we apply comparison, facilitation, competition to our design. We present a ranking table listing the progress of the members who have the same need or goal to the user so that they can aware of the situation of the others and compare the performance. And we also present other users' goals to the user who doesn't have the same goal. For example, "There are 20 members who are trying to achieve walking 10000 steps a day!". To achieve the competition function, in our design, the chatbot writes a periodical blog/report about the user who does the best performance. For example, "Weekly star, the man who walked most" and "Monthly star, the man who saved the most energy".

According to the theory of goal setting, people with high self-efficacy set higher goals and have higher performance than people with low self-efficacy [8]. Therefore, it's necessary to distinguish users' type and deal with them based on the type. In this design, users will be divided into several groups based on their needs, goals, past performance and current progress. And people who have high self-efficacy can set their own goals while the low self-efficacy people need to select the goals provided by the system.

Gamification may be effective for improving user motivation, and indeed it overlaps with our design with regards to multiple strategies such as social competition and comparison. However, because gamification combines a set of persuasion strategies with a playful user interface, in the case of achieved behavior change it still would be difficult to differentiate whether it resulted from playfulness or from the persuasion. Therefore, we decided not to import any more gamification elements into our design for this time.

\section{SUMmARY}

In this paper, we discussed the limitations of current behavior change support systems. To offset the limitations, we proposed a design of an environment for BCSSs in which we used an interactive signage with active talk function. There are four parts in the design and we seek to achieve the goal of forming, altering and reinforcing users' attitude or behavior by estimating users' needs and tracking users' progress. In order to improve the efficiency of digital interventions and the quality of behavior change, we picked up a set of persuasive software features with the help of the BCSS theory and explained how we apply them to our design. In the future, we are going to actually build this environment and run an experiment in a real-life setting to investigate how these features work in the environment.

\section{ACKNOWLEDGMENT}

This work is supported by JST PRESTO.

\section{REFERENCES}

[1] H. Oinas-Kukkonen, "A foundation for the study of behavior change support systems," Personal and ubiquitous computing, vol. 17, no. 6, pp. 1223-1235, 2013.

[2] S. Sankaran, I. Frederix, M. Haesen, P. Dendale, K. Luyten, and K. Coninx, "A grounded approach for applying behavior change techniques in mobile cardiac tele-rehabilitation," in Proceedings of the 9th ACM International Conference on PErvasive Technologies Related to Assistive Environments. ACM, 2016, p. 63.

[3] V. Strecher, "Internet methods for delivering behavioral and healthrelated interventions (ehealth)," Annu. Rev. Clin. Psychol., vol. 3, pp. 53-76, 2007.

[4] E. Elenko, L. Underwood, and D. Zohar, "Defining digital medicine," Nature biotechnology, vol. 33, no. 5, p. 456, 2015.

[5] T. Kari, J. Arjoranta, and M. Salo, "Behavior change types with pokémon go," in Proceedings of the 12th International Conference on the Foundations of Digital Games. ACM, 2017, p. 33.

[6] S. Consolvo, D. W. McDonald, and J. A. Landay, "Theory-driven design strategies for technologies that support behavior change in everyday life," in Proceedings of the SIGCHI conference on human factors in computing systems. ACM, 2009, pp. 405-414.

[7] J. Fortmann, V. Cobus, W. Heuten, and S. Boll, "Waterjewel: design and evaluation of a bracelet to promote a better drinking behaviour," in Proceedings of the 13th international conference on mobile and ubiquitous multimedia. ACM, 2014, pp. 58-67.

[8] E. A. Locke and G. P. Latham, "Building a practically useful theory of goal setting and task motivation: A 35-year odyssey." American psychologist, vol. 57, no. 9, p. 705, 2002.

[9] H. Oinas-Kukkonen and M. Harjumaa, "Persuasive systems design: Key issues, process model, and system features." Communications of the Association for Information Systems, vol. 24, 2009.

[10] N. Jaques, T. Rich, K. Dinakar, N. Farve, W. Chen, P. Maes, R. Picard, and K. Slavin, "Bitxbit: Encouraging behavior change with $n=2$ experiments," in Proceedings of the 2016 CHI Conference Extended Abstracts on Human Factors in Computing Systems. ACM, 2016, pp. 2134-2140.

[11] E. T. Luhanga, A. A. E. Hippocrate, H. Suwa, Y. Arakawa, and K. Yasumoto, "Identifying and evaluating user requirements for smartphone group fitness applications," IEEE Access, vol. 6, pp. 3256-3269, 2018

[12] J. Matthews, K. T. Win, H. Oinas-Kukkonen, and M. Freeman, "Persuasive technology in mobile applications promoting physical activity: a systematic review," Journal of medical systems, vol. 40, no. 3, p. 72 , 2016.

[13] P. Karppinen, H. Oinas-Kukkonen, T. Alahäivälä, T. Jokelainen, A.-M. Keränen, T. Salonurmi, and M. Savolainen, "Persuasive user experiences of a health behavior change support system: A 12-month study for prevention of metabolic syndrome," International journal of medical informatics, vol. 96, pp. 51-61, 2016.

[14] K. Stawarz, A. L. Cox, and A. Blandford, "Beyond self-tracking and reminders: designing smartphone apps that support habit formation," in Proceedings of the 33rd annual ACM conference on human factors in computing systems. ACM, 2015, pp. 2653-2662.

[15] Z. Zhang, Y. Takahashi, M. Fujimoto, Y. Arakawa, and K. Yasumoto, "Investigating user reactions to interactive-signage-based stimulation toward behavior change," in The 11th International Conference on Mobile Computing and Ubiquitous Networking (ICMU2018), Oct. 2018.

[16] T. Alahäivälä and H. Oinas-Kukkonen, "Understanding persuasion contexts in health gamification: A systematic analysis of gamified health behavior change support systems literature," International journal of medical informatics, vol. 96, pp. 62-70, 2016. 